NASA Technical Memorandum 101547

\title{
Some Low-Speed Flutter Characteristcs of Simple Low-Aspect-Ratio Delta Wing Models
}

Robert V. Doggett, Jr. and David L. Soistmann

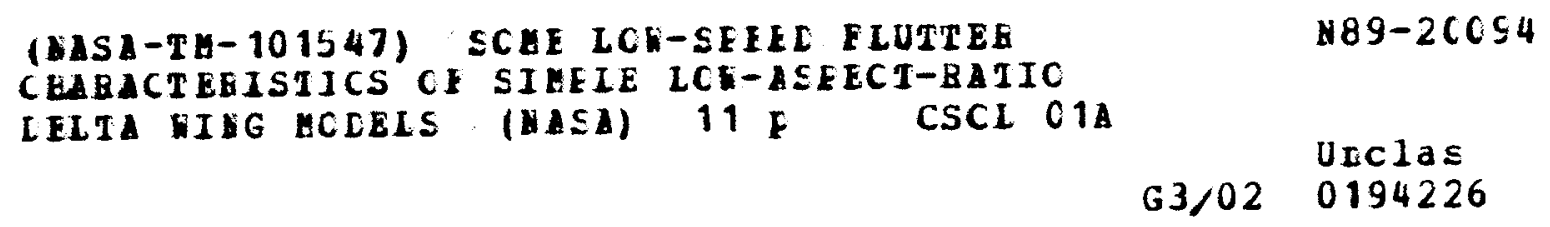

January 1989

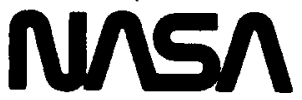

National Aeronautics and

Space Administration

Langley Research Center

Hampton, Virginia 23665-5225 


\title{
SOME LOW-SPEED FLUTTER CHARACTERISTICS OF SIMPLE LOW-ASPECT-RATIO DELTA WING MODELS
}

\author{
Robert V. Doggett, Jr." \\ NASA Langley Research Center \\ Hampton, Virginia \\ and \\ David L. Soistmann+ \\ Planning Research Corporation \\ Hampton, Virginia
}

\begin{abstract}
Some results from a combined experimental and analytical study of the low-speed flutter characteristics of low-aspect-ratio delta wings are presented. Data are presented which show the effects of sweep angle on the flutter characteristics of some simple plate models of constant planform area. The range of sweep angles studied was from 30 to 72 degrees. In addition, flutter results are presented for two 300-sweep clipped-delta wing models. Further, results are presented that show the effects of root clamping (percentage length of the root chord that is cantilevered) for a 450-sweep delta wing. The experimental data are compared with analytical results obtained by using kernel function and doublet lattice subsonic unsteady lifting surface theories.
\end{abstract}

\section{Introduction}

Rekindled interest in hypersonic aircraft has resulted in the need to better understand the flutter characteristics of low-aspectratio delta wing configurations which are candidate planforms for use with such airplanes. In his excellent summary of experimental and theoretical flutter studies1, Harris observed that "more difficulty was experienced in obtaining data ... for surfaces of delta type planform than was the case for conventional planforms." Although this observation was made in 1963, the situation is not much different today. A recent literature survey by Reed et al2 focused on the flutter characteristics of candidate hypersonic airplane configurations. They identified only tive papers published since 1963 that present experimental flutter data for delta wings.

"Chief Scientist, Structural Dynamics Division, Associate Fellow AIAA, Member AHS.

+Structures Engineer, Member AIAA.
The current study, part of a much larger effort to provide a better understanding of delta wing flutter characteristics throughout the Mach number range, was undertaken to determine the low-speed flutter characteristics of some simple delta wing configurations. The primary parameters studied were leading edge sweep, varying from 30 to 72 degrees: percentage removed from the tip of a 300 . sweep delta wing to form clipped delta wings, 20 and 30 percent; and percentage length along the root chord that was clamped (cantilevered), varying from 21 to 100 percent, for a 450-sweep delta wing. The experimental results were obtained in the NASA Langley Research Center Transonic Dynamics Tunnel. Analytical results obtained by using kernel function and doublet lattice subsonic unsteady lifting surface theories were compared with the experimental results.

\section{Description \\ Models}

There were three series of model configurations tested. The first series, hereafter referred to as sweep-effects series, consisted of four delta-wing configurations of constant 260 in.2 planform area with respective leading edge sweep angles of 300,450 , 600 and 720 and corresponding panel aspect ratios of $3.45,2.00,1.15$, and 0.65 . Sketches of these configurations are shown in Fig. 1. The models were constructed of aluminum alloy plate. The plate thickness for the 300 . sweep model was 0.091 in.; the plate thickness for the 600-sweep model was 0.064 in.; the plate thickness for the 720-sweep model was $0.051 \mathrm{in}$. There were two models with 450 sweep. The plate thicknesses for these two models were 0.064 in. and 0.091 in. 

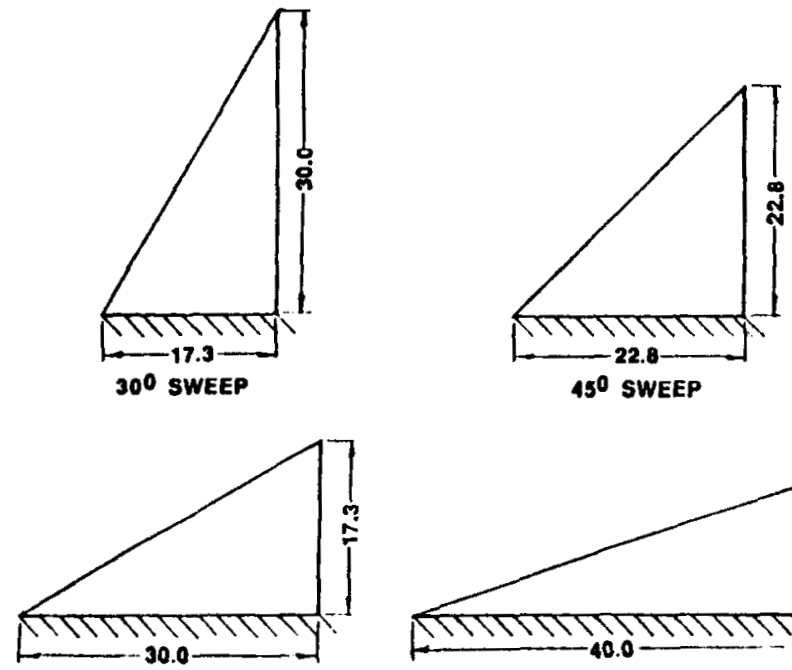

100 SWEEP

Fig. 1 Geometry of sweep-effects models. (Linear dimensions are in inches.)

The second series of configurations, hereafter referred to as the tip-clippingeffects series, consisted of a $300-s w e e p$ delta wing configuration and two variations thereof. These variations in configuration were clipped delta wings. For one, the outermost 20 percent of the basic delta wing was removed; for the other, the outermost 30 percent of the basic delta wing was removed. The respective planform areas of these three models were 260 in.2, 250 in.2, and 237 in.2 with corresponding panel aspect ratios of 3.45 , 2.31, and 1.87. These models were constructed of 0.091 in.-thick aluminum alloy plate. Sketches of these configurations are presented in Fig. 2.
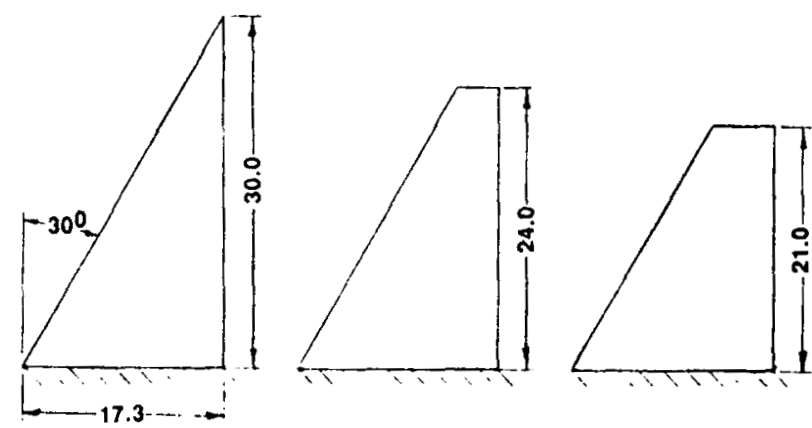

Fig. 2 Geometry of tip-clipping-effects models. (Linear dimensions are in inches.)

The third series of configurations, hereafter referred to as the root-clamping-effects series, consisted of variations in the percentage length of the root chord that was clamped (cantilevered) for a 450-sweep delta wing having a planform area of 260 in. 2 and a panel aspect ratio of 2.00. The clamping was symmetric about the center of the root chord of the models. This model was constructed from 0.064 in.-thick aluminum alloy sheet. This model was serially modified by sawing along the root chord so that the length of the cantilever root was reduced first from 22.8 inches (100-percent root chord) to 16.8 inches (74percent root chord), then reduced to 10.8 inches (47-percent root chord), next reduced to 7.8 inches (34-percent root chord), and finally reduced to 4.8 inches (21-percent root chord). An equal amount was sawed from the leading and trailing edges so that the clamping was symmetric about the center of the root chord. Sketches of each modification of the model are shown in Fig. 3. The model was flutter tested after each modification.

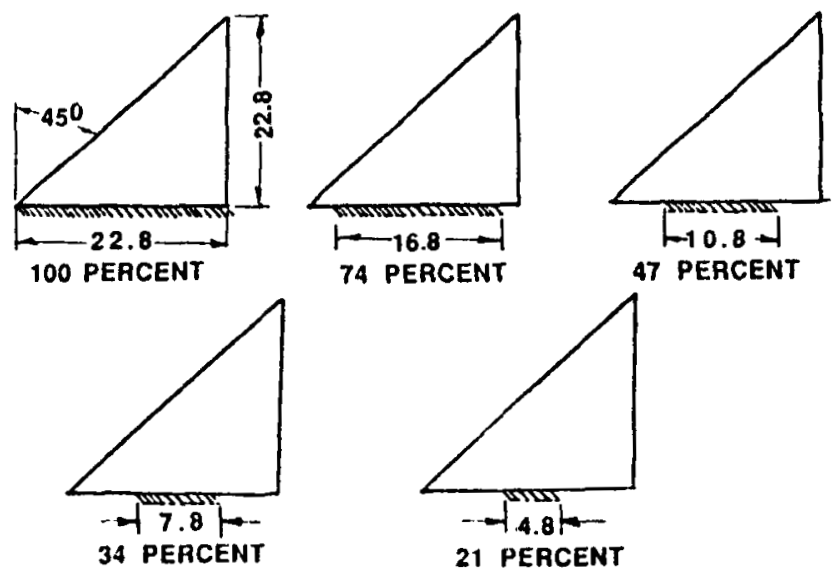

Fig. 3 Geometry of root-clamping-effects models. Clamping is symmetric about center line of root chord. (Linear dimensions are in inches.)

For all the models the leading and trailing edges of the aluminum plate were beveled to form sharp edges. Each model was instrumented with two four-arm resistance-wire strain gage bridges. One bridge was oriented to be primarily sensitive to strains produced by spanwise bending deflections whereas the other bridge was oriented to be primarily sensitive to strains produced by torsional, or chordwise, deformations. In addition, transition strips of \#30 carborundum grit were installed on the upper and lower surfaces of all models. The center of each strip was at 10 percent of the local chord; the streamwise width of each strip was about two percent of the local chord. 


\section{Vibration Characteristics}

The natural vibration frequencies, mode shapes and generalized masses were calculated. To accomplish this a finite-element model was developed for each model configuration tested by using the EISI-EAL Engineering Analysis computer code 3 . The wings were modeled using a combination of triangular and quadrilateral plate elements. A typical arrangement of finite elements is shown in Fig. 4.

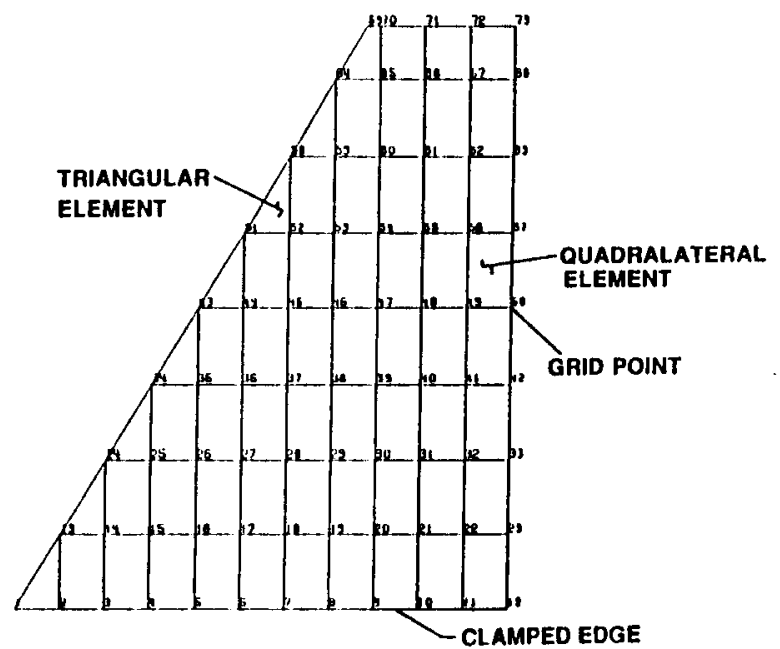

Fig. 4 Typical structural finite-element model.

The first five natural frequencies and corresponding nodal patterns were measured for most of the models. To obtain these data the models were excited by using a variable frequency pulsating air jet. The nodal patterns were obtained by observing the gravitation of sand sprinkled on the surface of the vibrating models to the node lines, points of no vibration.
Sweep-effects series. Calculated nodal patterns are presented in Fig. 5. Although they are not shown in the figure, the measured nodal patterns were very similar to the calculated ones. An examination of the nodal patterns in Fig. 5 shows that the patterns for the 300-sweep delta wing model exhibit a typical set of what is usually termed bending and torsion modes. For example, the first, second, and fourth modes are the first, second, and third bending modes. The third and fifth modes are first and second torsion modes. The sixth and seventh modes are coupled bending-torsion modes. However, as the model sweep angle increases the node lines change considerably and the classification of modes into bending and torsion categories is not as obvious. For example, the second mode for the 720-sweep wing appears to be a torsion mode from the location of the node line, but a careful examination of the "migration" of nodal patterns as sweep angle is increased shows that this mode is actually the modal equivalent of the second bending mode for the 300-sweep model, that the third mode for the 720-sweep wing is the modal equivalent of the second bending mode (fourth mode) of the 300 -sweep wing, and that the fourth mode for the 720 -sweep wing is the modal equivalent of the first torsion mode (third mode) of the 300 -sweep wing.

Some calculated and measured natural frequencies are presented in Fig. 6. The curves in these figures are fared through the calculated results. The measured frequencies are differentiated from the calculated ones by the use of ticked symbols. For presentation in this figure the frequencies have been
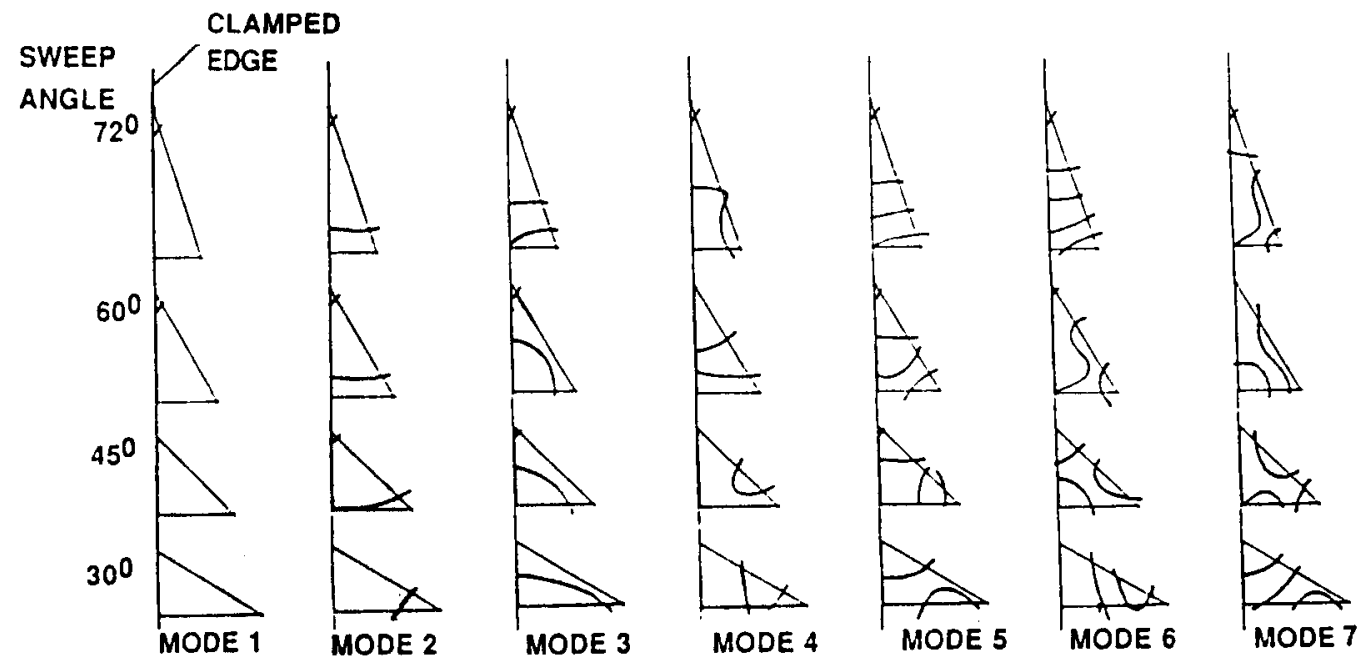

Fig. 5 Calculated node lines for sweep-effects models. 


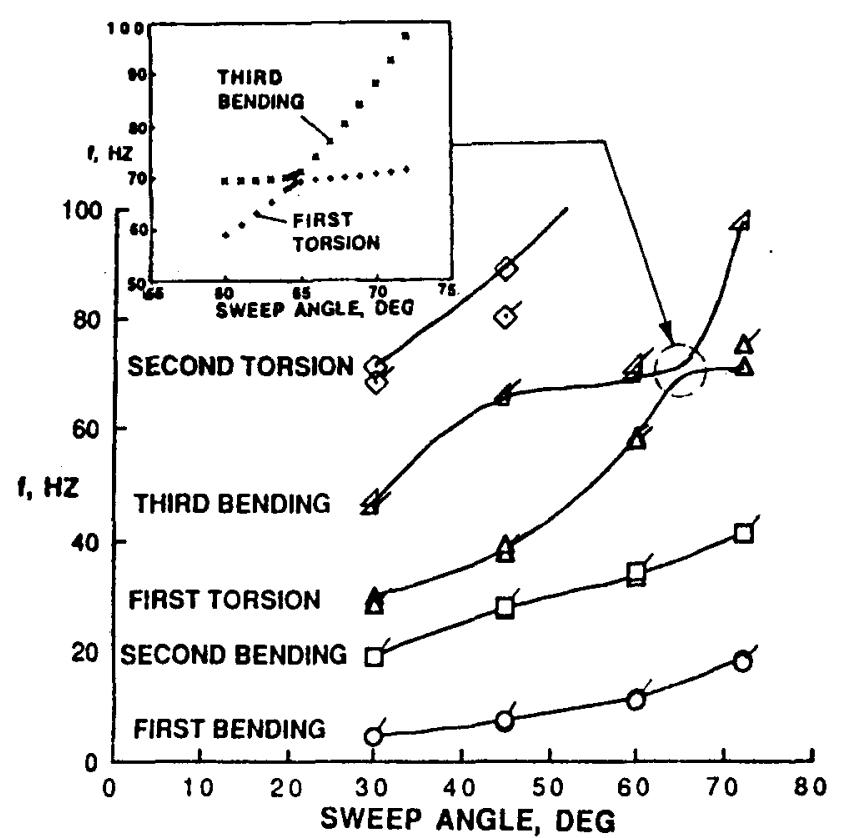

Fig. 6 Cakculated and measured natural frequencies of sweep-effecls models. (Frequencies adjusted for plate thickness $=0.064 \mathrm{in}$.)

adjusted to the values they would have if all the wings were 0.064 in. thick by using ratios of the actual plate thickness to the reference thickness. The measured frequencies are in good agreement with the calculated frequencies. The variations of the modal frequencies with sweep angle are easily seen. Although the curves for the modes labeled first torsion and third bending do no: actually cross as shown by the inset in Fig. 6 , they do come very close together. As the curves approach one another as sweep angle is increased, they change their direction so that one appears to be the extension of the other which results in a switching of character of the two modes from one side of the "crossing point" to the other as indicated by the nodal patterns shown in Fig. 5. The vibration results shown here are consistent with the vibration characteristics of platelike delta wings observed by other investigators 4,5

Iip-Clipping-Effects Series. Calculated node lines and calculated and measured natural frequencies are presented in Figs. 7 and 8 , respectively. The actual frequencies are presented in Fig. 8 because the thickness was the same for all models in this series. There is no switching of the character of modes in the frequency spectra for these models over the range of tip clipping studied although the frequencies of the second bending and first torsion modes tend to approach each other as more of the tip is clipped. The calculated and experimental frequencies are in good agreement. Although not shown, similar agreement was found between calculated and measured nodal patterns.

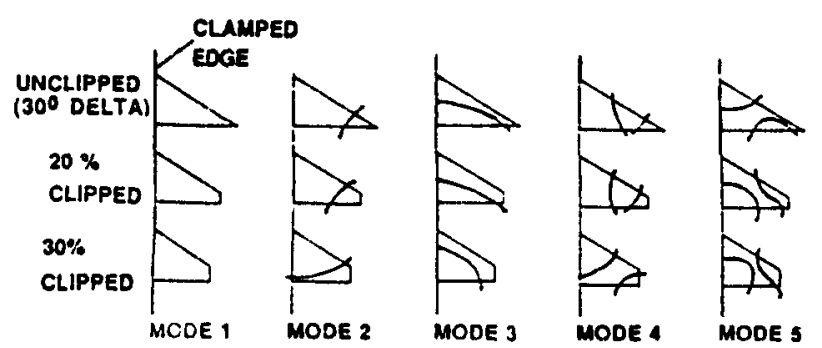

Fig. 7 Calculated node lines for clippedtip-effects moduls

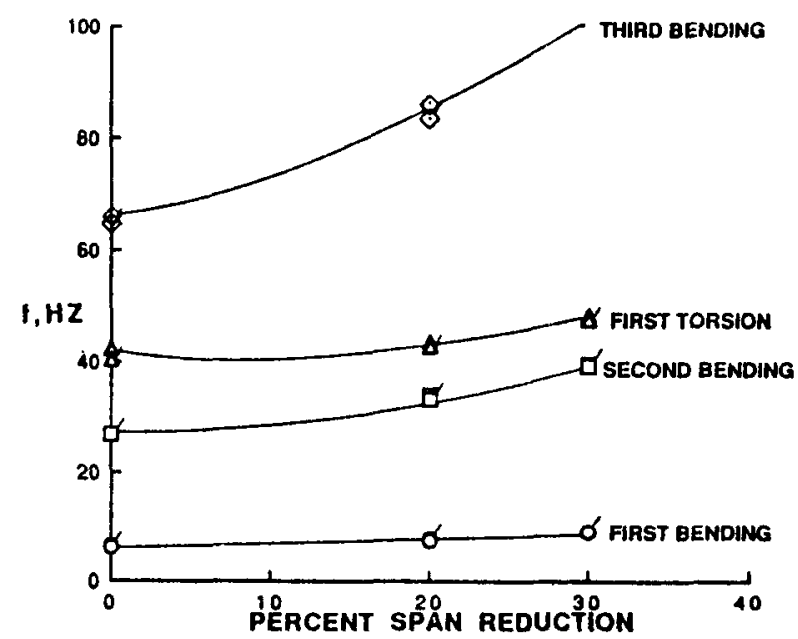

Fig. 8 Calculaled and measured natural frequencies for clipped-tip-effects models.

Boot-Clamping-Effects Series, Calculated nodal patterns are presented in Fig. 9. Although not shown, the measured nodal patterns were very similar to the calculated ones. Some calculated and measured natural frequencies are presented in Fig. 10. The actual frequencies are shown in this figure because the models in each case had the same thickness. The agreement between the measured and calculated frequencies is good. The data for this series of models

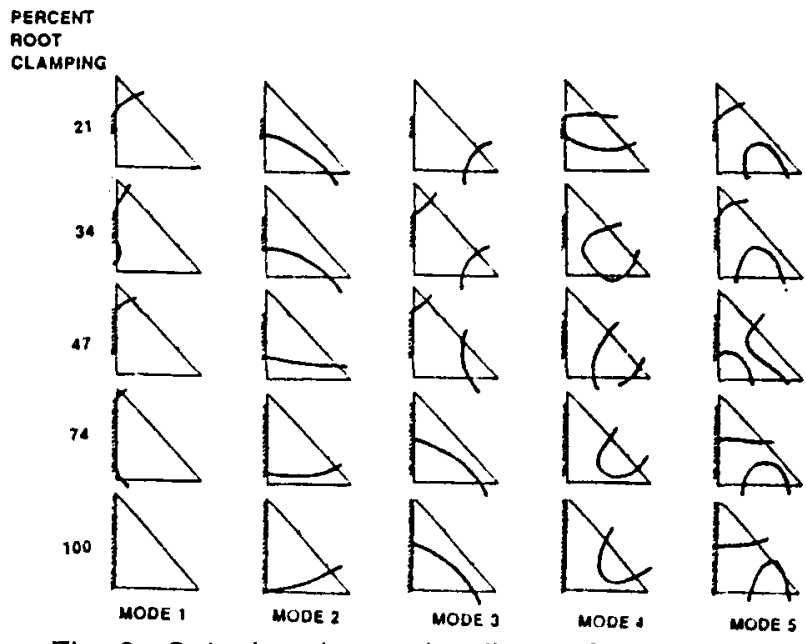

Fig. 9 Calculated node lines for rootclamping-effects models. 
also show a switching of bending and torsion modes in the order of natural modes similar to that described previously for the sweepeffects series. In this case, as percent root clamping is decreased, the curves for the modes labeled second bending and first torsion come close together at about 45-percent root clamping. A comparison of the node line for the third mode of the 100-percent-clamped model with the second mode of the 21-percent clamped model shows little difference. Therefore the second mode of the latter (21. percent) is the modal equivalent of the third mode of the former (100-percent).

The overall good agreement between calculated and measured vibration characteristics for all three series of models validated the accuracy of the analytical finite-element model.

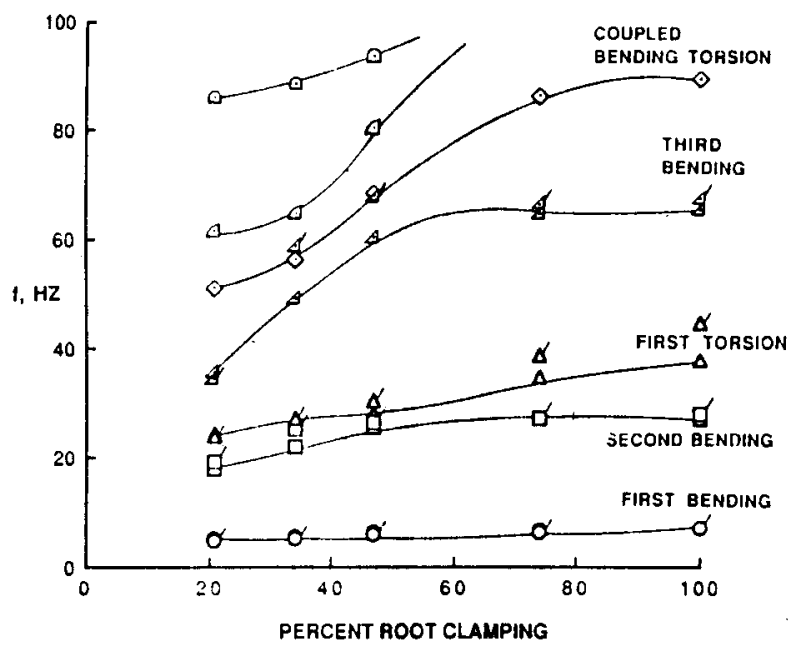

Fig. 10 Calculated and measured natural frequencies of root-clampingeffects models.

\section{Wind Tunnel}

\section{Flutter Experiments}

The wind-tunnel tests were conducted in the Langley Transonic Dynamics Tunnel (TDT). This wind tunnel is used almost exclusively for aeroelastic testing. The TDT is of the single return type, and its speed and stagnation pressure are continuously controllable over a range of Mach numbers from near zero to 1.2 and a range of pressures from near vacuum to about one atmosphere. Either air or a heavy gas $(R-12)$ can be used as the test medium. Air at atmospheric stagnation pressure was used for the present test.

\section{Elutter Test Procedure}

The determination of a flutter point proceeded as follows: With the tunnel stagnation pressure set at atmospheric pressure, the fan rpm was gradually increased in steps and the model response was observed visually by the test engineer. The output of the strain gage bridges were monitored by another engineer on a strip chart recorder. In addition, the mean values of the strain gage signals, proportional to static load, were displayed on a digital display. When the observations indicated that a flutter condition had been reached, the windtunnel flow conditions were recorded after which the tunnel speed was rapidly reduced. In addition, the strain gage output signals were monitored using a transfer function analyzer to obtain the predominant frequency content of the model response and to monitor the response of the model as flutter was approached.

For testing, the models were clamped in a set of blocks, cantilever root condition, that was in turn attached to a remotely controlled lurntable on the wall so that the angle of attack could be changed during testing. The model attitude was continuously adjusted to near zero lift by using the turntable. The mounting arrangement was such that the root chord of the model was outside the windtunnel wall boundary layer.

Natural frequencies of each model were checked before and after each flutter point to ensure that the model had not been damaged. No damage was detected.

\section{Flutter Analysis}

Flutter calculations were made by using kernel function 6 and doublet lattice 7 subsonic unsteady lifting surface theories. Typically, 48 collocation points, eight chordwise points at each of six spanwise stations, were used for the kernel function calculations. The number of doublet lattice boxes varied from 75 to 140 with the total number of boxes and the number of boxes per chord increasing with increasing model sweep angle. The aspect ratios of the doublet lattice boxes were for the most part close to one. Illustrative arrangements of collocation points and doublet lattice boxes are shown in Fig. 11. (This is the same model for which the finiteelement model was shown in Fig. 4.) The first seven calculated mode shapes, generalized masses, and natural ,frequencies were used in the analyses. A structural damping ratio of 0.01 was used for all modes. A $p-k$ flutter solution method 8 was used to solve the flutter 


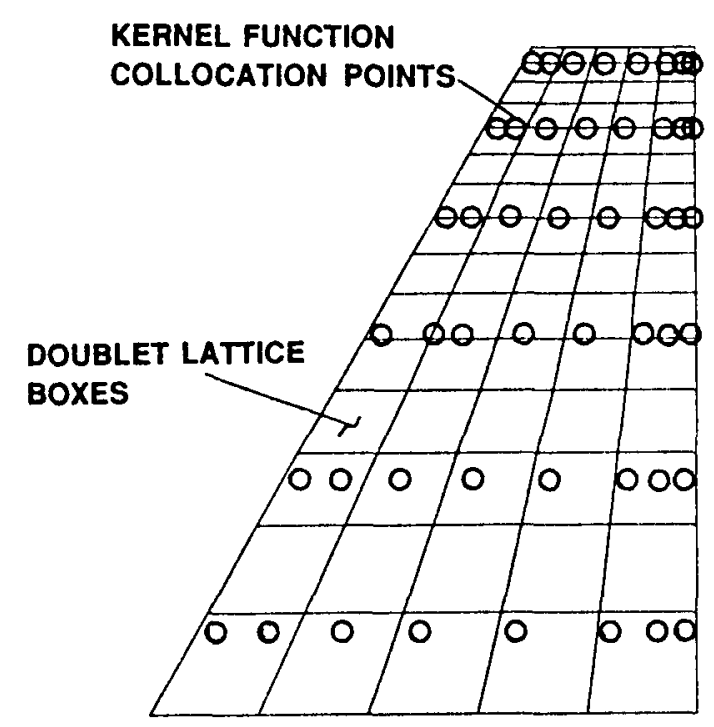

Fig. 11 Typical arrangement of doublet lattice boxes and kernel function collocation points.

equations and the results were plotted in a root-locus format. A typical result is shown in Fig. 12.

The calculations were made at Mach numbers corresponding to the experimental values. The calculated results are "matched points" in that the flutter speed is consistent with the Mach number for which each individual calculation was made.

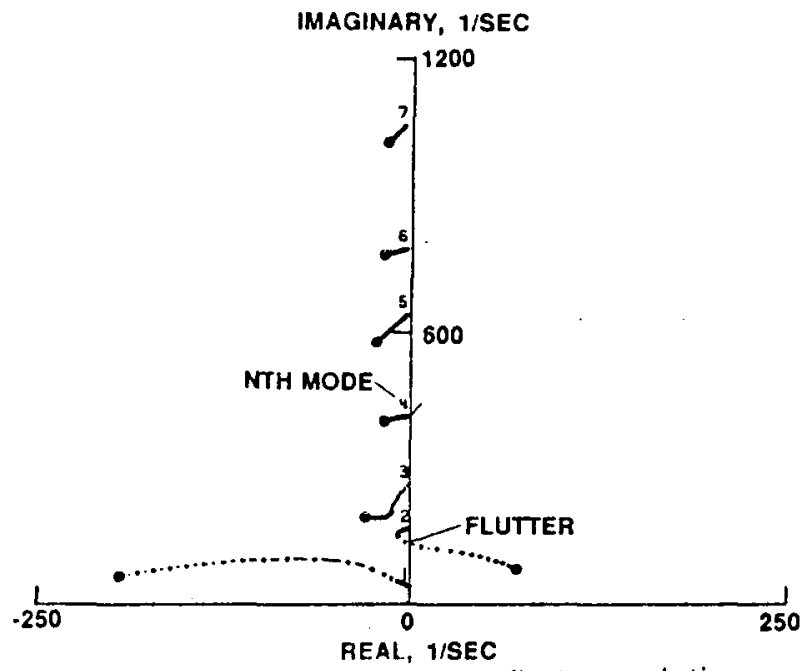

Fig. 12 Typical root locus flutter solution.

\section{Results and Discussion}

The flutter results are presented in the form of the flutter velocity index $V_{1}$ which is a parameter commonly used to correlate flutter data from different configurations. This parameter is defined by the relationship

$$
V_{1}=\frac{V}{2 \pi f_{r e t} b_{r e l} \sqrt{\mu}}
$$

where $V$ is the flutter speed; bref is a reference length (mean semichord was used here); fret is a reference frequency, usually the torsion natural frequency which was used here; and $\mu$ is the mass ratio parameter. The mass ratio parameter is defined as the mass of the wing divided by the mass of a circumscribed volume of air. For the delta wing models the volume used was that contained in a right circular cone that had a base diameter equal to the wing root chord and an altitude equal to the wing span. For the clipped delta wings, the volume was that of a conical frustum having diameters equal to the wing root and tip chords and an altitude equal to the wing span. The mass of the fluid is the fluid density at the flutter condition multiplied by this volume.

Because the experimental flutter results were obtained at very low speeds (the Mach number range was from about 0.10 to about $0.35)$, compressibility effects are assumed to be very small and the data are presented and discussed as if they were all obtained at the same Mach number. Recall that the calculations were made at Mach numbers corresponding to the experimental results.

The flutter experimental and calculated results are presented in Figs.13-15. Presented at the top of each figure is the variation of the ratio of flutter frequency to natural torsion frequency with the parameter being studied, for example, sweep angle. Presented at the bottom of each figure is the variation of the flutter velocity index with the parameter. The experimental results are indicated by the symbol and solid line faring. The doublet lattice calculated results are marked by the dashed line. The kernel function results are indicated by the long/short dashed line.

Sweep-angle ellects

The results for the sweep-effects series are presented in Fig. 13. The experimental frequency results show that the flutter frequency as a fraction of the torsion increases slightly as sweep angle is increased from 30 to 45 degrees and then begins to decrease in value as sweep angle increases further. The flutter frequency ratio ranges from about 40 to 60 percent of the torsion frequency over the range of sweep angles investigated. The flutter velocity index shows a linear decrease in value as sweep angle is increased from 30 to 72 degrees. The $V_{1}$ parameter does an excellent job of correlating the two flutter points obtained for the two 450 -sweep mod- 
els. There was almost a factor of three difference in actual flutter dynamic pressures for these models.

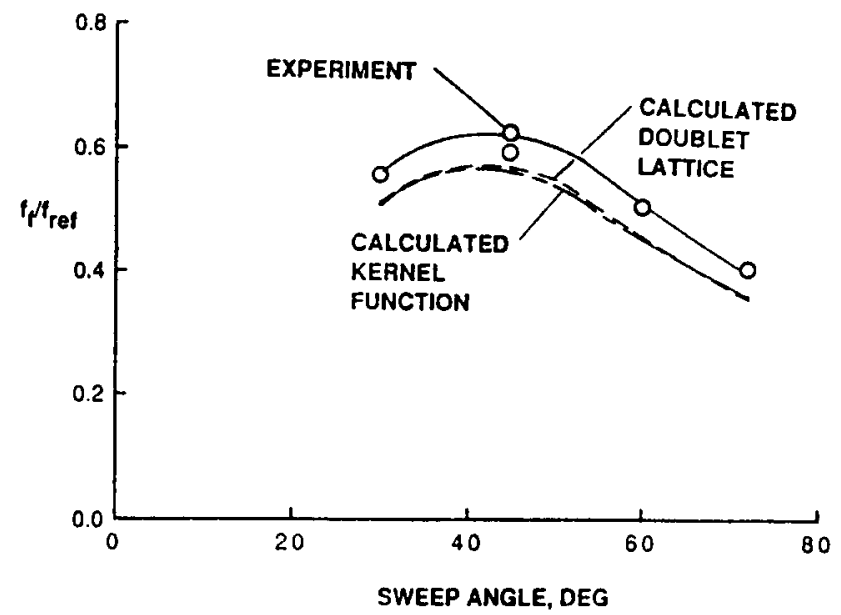

(a) Flutter frequency results.

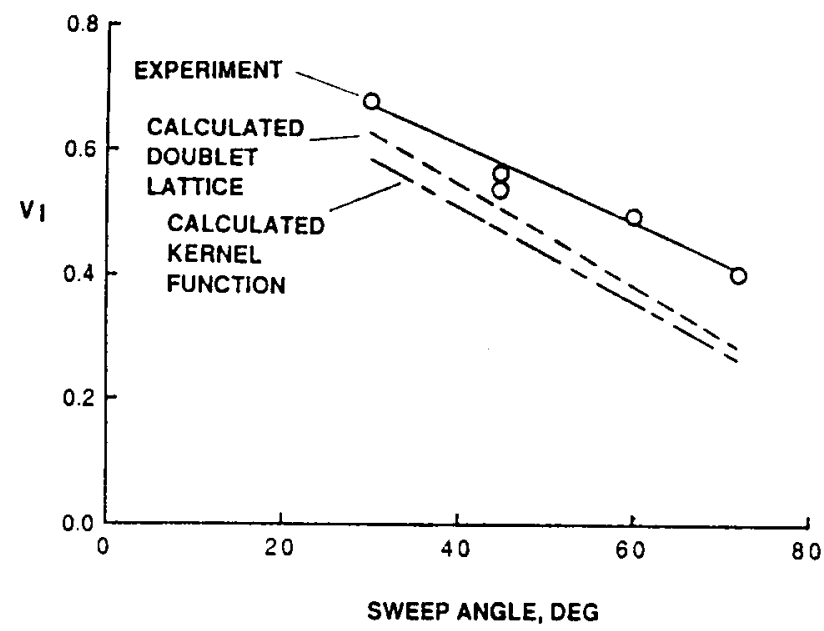

(b) Flutter velocity results.

Fig. 13 Variation of flutter frequency ratio and flutter velocity index with sweep angle.

A few comments about the nature of the flutter that was observed are in order. For the lower sweep angles flutter onset was clear in that at flutter the response began to increase at a rapid rate in the usual divergent amplilude oscillation. As sweep angle was increased, however, the onset of flutter became less distinct. As the flutter condition was approached the higher sweep models exhibited long bursts of lowly damped oscillations. The flutter condition itself was in the nature of a limited amplitude oscillation.

The trends of the calculated frequency and velocily results are very similar to the experimental results. The calculated frequency results obtained by using the two theories were essentially the same. The calcu- lated flutter velocity boundary is conservative with respect to the experimental results. The disagreement between theory and experiment increases with increasing sweep angle.

Iip-clipping effects

The results for the 300 -sweep clipped delta wings are presented in Fig. 14. The ratio of experimental flutter frequency to torsion frequency is almost constant, about 0.55 in value, over the range of tip clipping studied. The experimental flutter speed as indicated by the $V_{\mid}$parameter decreases as tip clipping is increased from the pointed tip delta wing to a 30-percent clipped-delta wing.

The trends of the calculated frequency and velocity results are very similar to the experimental results. The calculated frequency results obtained by using the two theories are very close in value. Both sets of calculated flutter velocity boundaries are in good

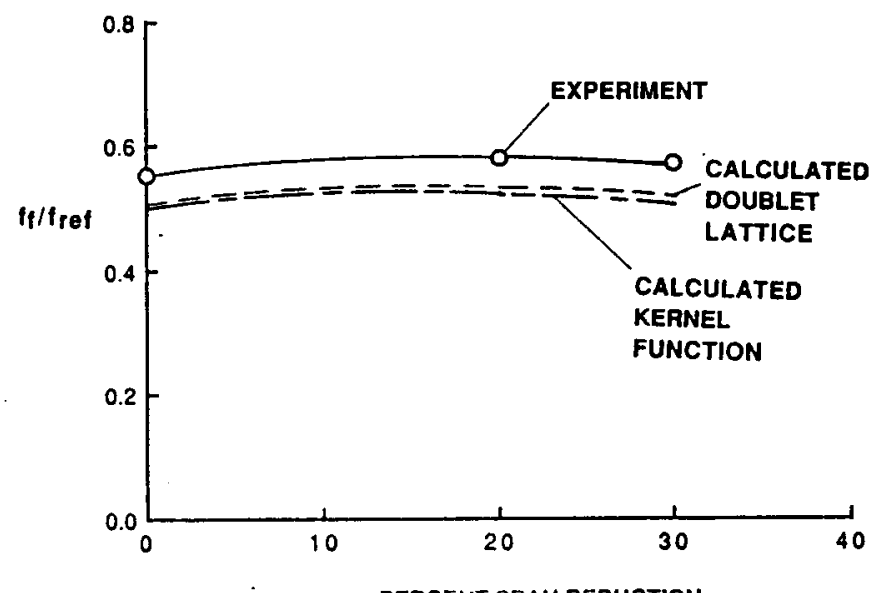

(a) Flutter frequency results.

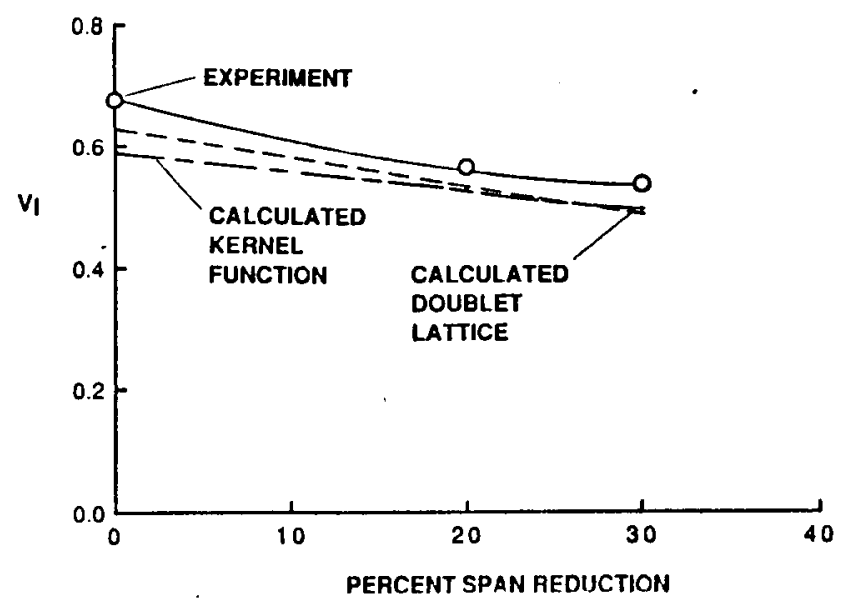

(b) Flutter velocity results.

Fig. 14. Variation of flutter frequency ratio and flutter velocity index with percent span reduction for 300 . sweep delta wing. 
agreement with one another and are conservalive with respect to the experimental results. The agreement between theory and experiment appears to improve slightly as tip clipping increases.

\section{Root-clamoing effects}

The results for the root-clampingeffects series of models are presented in Fig. 15. The experimental flutter frequency ratio results show a saddle at about 35percent root clamping, indicating a transition from flutter of one characteristic to that of another characteristic. The reason for this is believed to be due to a change in relative

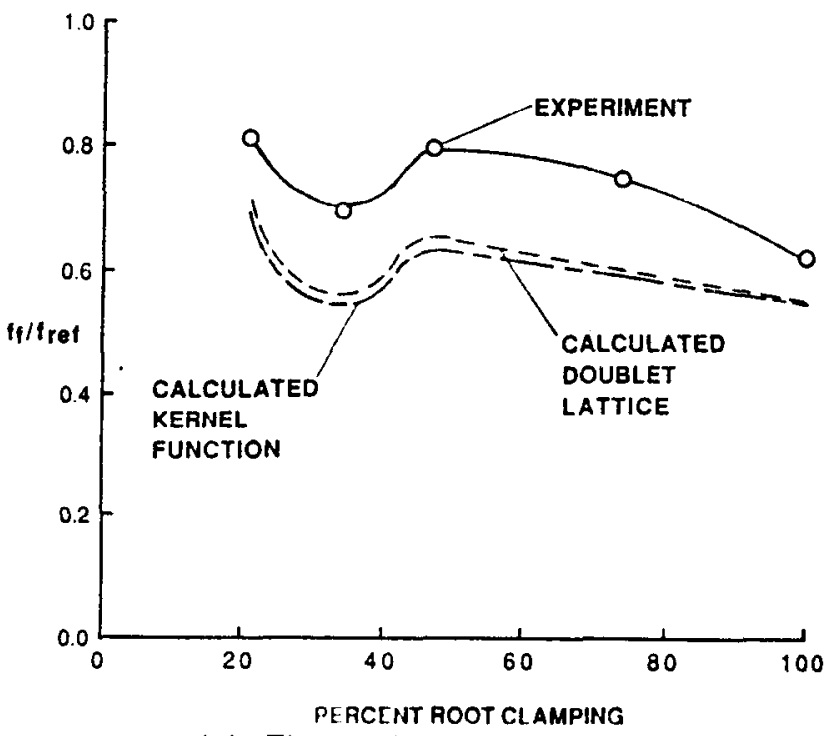

(a) Flutter frequency results.

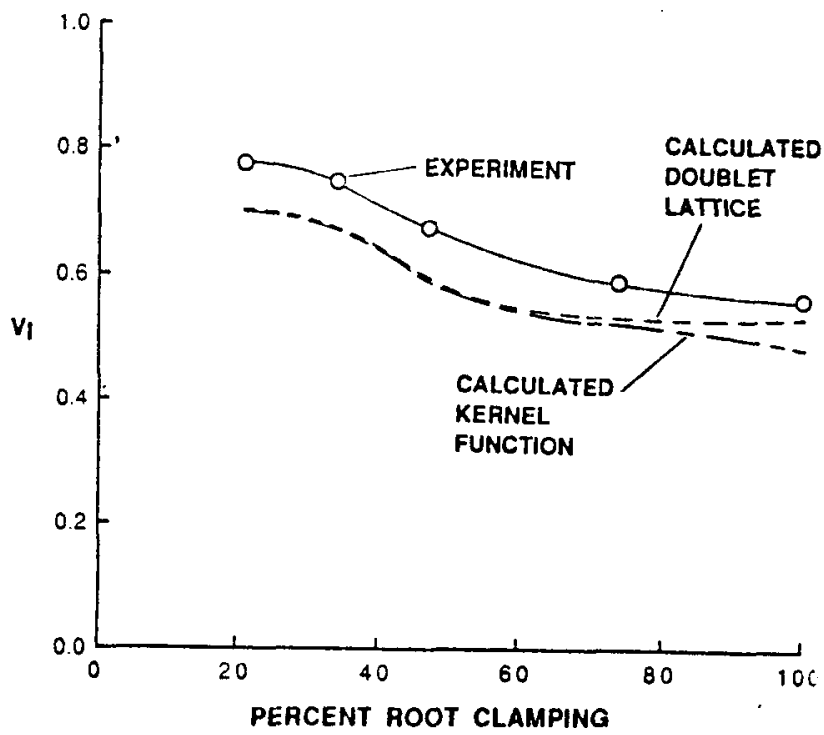

(b) Flutter velocity results.

F Fig. 15. Variation of flutter frequency ratio and flutter velocity index with percent root clamping for 450 . sweep delta wing. participation of the bending and torsion natural modes in the flutter mode. Recall that the second and third natural modes, second bending and first torsion, switch places in the frequency spectrum near a root clamping value of about 45 percent (see Fig. 8). The calculated flutter roots are associated with the second natural frequency. The flutter velocity index results show a decrease in flutter speed as the percentage of the root that is clamped is increased.

The trends of the calculated frequency results are very similar to the experimental results, exhibiting the same saddle shape as discussed previously. The calculated frequency results obtained by using the two theories are very close in value. Both sets of calculated flutter velocity boundaries are in good agreement with one another, almost identical in value for the smaller values of root clamping, and are conservative with respect to the experimental results.

\section{Concluding Remarks}

Experimental and analytical flutter results have been presented for some lowaspect-ratio delta wings with sweep angles ranging from 300 to $72^{\circ}$. The study included the determination of the effects on flutter characteristics of leading edge sweep angle, of removing portions of the tip to form a clipped delta configuration for a 300 -sweep wing, and of variations in percentage of the root that was clamped (cantilevered) for a 450 sweep wing. The results show that for flutter speeds expressed in the form of the flutter velocity index, the flutter speed decreases with increasing sweep, decreases with decreasing span, and decreases as the percentage of the root that is clamped is increased. Analytical results obtained by using kernel function and doublet lattice subsonic unsteady lifting surface theories were always conservative and agreed better with the experimental results for the lower sweep angles. There were only small differences between the two analytical results.

\section{Beferences}

1. Harris, G., "Flutter Criteria for Preliminary Design," Bureau of Naval Weapons Contract NOw61-1072, Final Engineering Report 2-53450/3R467, LTV/Chance Vought Corp., Sept. 1963. 
2. Reed, W. H., III, Hanson, P. W., and Alford, W. J., Jr., "Assessment of Flutter Model Testing Relating to the National AeroSpace Plane," NASP Contractor Report 1002, July 1987.

3. Whetone, W., "EISI-EAL Engineering Analysis Language Reference Manual," Engineering Information Systems, Inc., San Jose, CA, 1983.

4. Gustafson, P. N., Stokey, W. F., Zorowski, C. F., "An Experimental Study of Natural Vibrations of Cantilevered Triangular Plates," J. of the Aero. Sci., Vol. 20, No. 5, pp.331-337, May 1953.

5. Leissa, A. W., "Vibration of Plates," NASA SP-160, 1969.

6. Cunningham, A. M., Jr., "A Steady and Oscillatory Kernel Function Method for Interfering Surfaces in Subsonic, Transonic and Supersonic Flow," NASA CR-144895, 1976.

7. Geising, J. P., Kalman, T. P., and Rodden, W. P., "Subsonic Unsteady Aerodynamics for General Configurations, Part I, Vol. IDirect Application of the Nonplanar Doublet-Lattice Method," AFFDL TR-71-5, Part I., Vol., I, 1971.

8. Adams, W. M., Jr., Tiffany, S. H., Newsom, J. R., and Peele, E. L., "STABCAR - A Program for Finding Characteristic Roots of Systems Having Transcendental Stability Matrices," NASA TP-2165, 1984. 


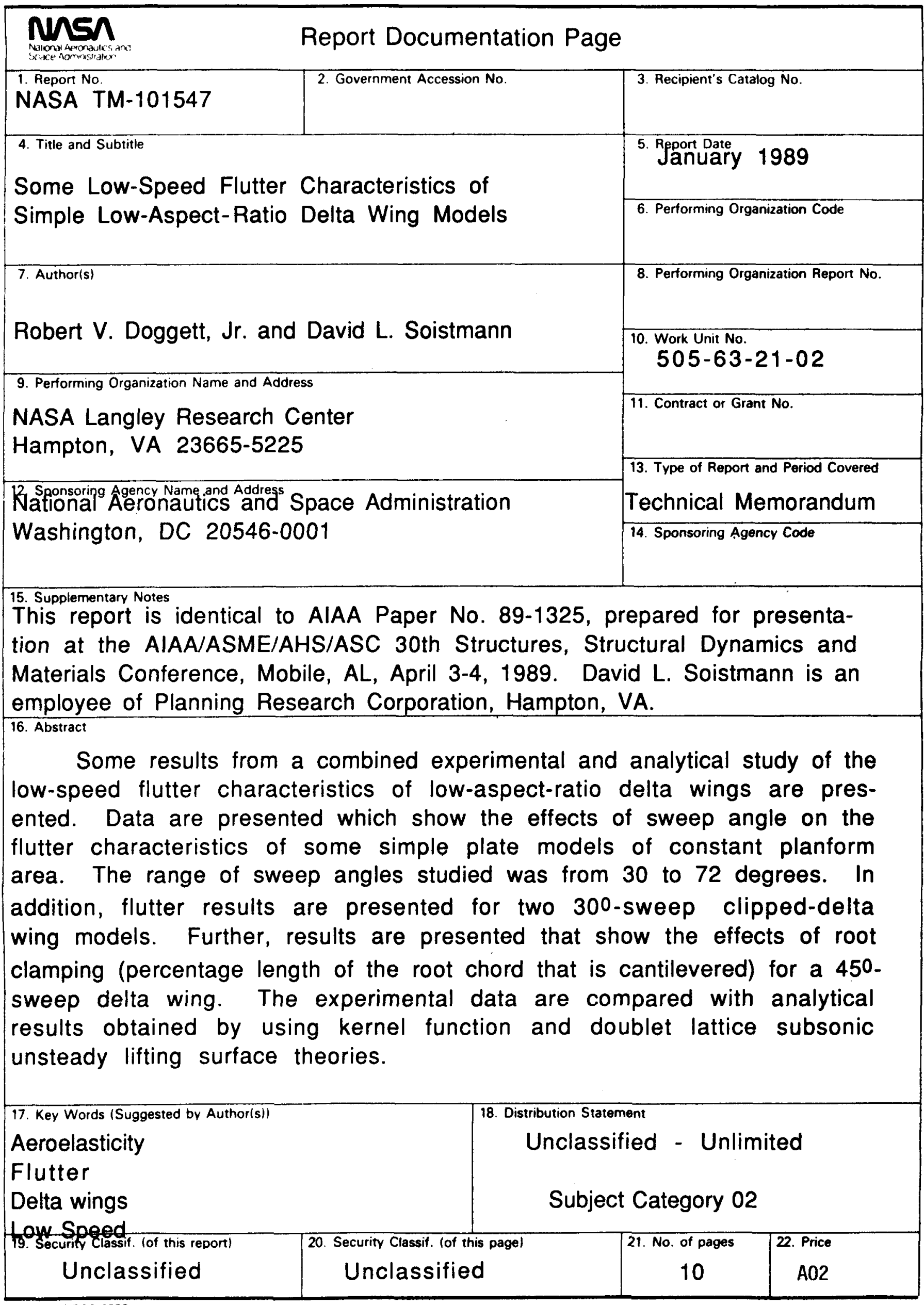

